\title{
Hybrid Three-Dimensional Reconstruction Methodology for Obtaining Structural Information of Locally Ordered Connexin26 Gap Junction Lattices.
}

\author{
G.E. Sosinsky* and S.J. Ludtke**
}

* National Center for Microscopy and Imaging Research, University of California, San Diego, 9500 Gilman Drive, La Jolla, CA 92093-0608 and **National Center for Macromolecular Imaging, Baylor College of Medicine, One Baylor Plaza Houston, TX 77030.

One type of cell-cell contact is a gap junction. Gap junctions are critical in coordinating responses, regulating cell ensembles, helping to synchronizing the action of neurons, transporting second messenger molecules and removing of secreted ions. The term "gap junction" refers to a cellular structure containing multiple intercellular channels spanning the two plasma membranes and the narrow extracellular "gap" that separates them. Gap junctions were one of the first specimens examined by electron crystallographic methods, however, the overwhelming majority of these in situ crystals have good short range order combined with long range disorder that results in coherent diffraction data only to about $\sim 15-25 \AA$ (as recently reviewed in [1]). As a result, the number of published 3D structures obtained at $>10 \AA$ is one [2]. Reconstitution into two-dimensional crystals is possible [3], however, we have found that there are classes of interesting mutants where the hexamer or dodecamer is stable when held within the membrane, but dissociate upon detergent solubilization [4]. For these mutants, crystallization for a high resolution crystal form will never be possible. In addition, we are using AFM as a tool for probing conformational changes that occur with treatments known to close gap junction channels [5] and want to correlate the AFM surface views with which part of the 3D structure moves during this conformational change. Therefore, we are working to reinvent image processing procedures that would allow us to obtain structural data from these locally ordered lattices.

Two reconstruction methods, single particle and tomographic reconstruction, hold promise for delivering $3 \mathrm{D}$ structural results that are not dependent on long-range lattice order. These approaches would be based on a combining 3D reconstruction methodology with crystallographic averaging prior to single particle averaging or crystallographic averaging after tomographic reconstruction. Recently, a new tomographic reconstruction algorithm shows great promise for higher resolution reconstruction [6]. Here, we describe our efforts in developing hybrid methods of image processing combining crystallographic information with single particle reconstruction methods. Our initial strategy for the correlation averaging follows that outlined in $[7,8]$ and uses projection matching to a model to calculate the 3D Euler angles that allow for accurate back projection of the images to a $3 \mathrm{D}$ structure. For our trial experiments, we used our hemichannel reconstruction [9] and original image data to test this processing scheme. This data set has two advantages: 1) the 41 images contain tilt data between $\sim \pm 60^{\circ}$ and approximates a good range of Euler angle values and 2) a structure that we can compare differences in structural features between the two methods. The initial alignment model used was either the crystallographically determined structure or a generalized model of a six plus one sphere arrangement. As is common in single particle reconstruction, subsequent cycles of refinement used the average from the previous cycle as the reference.

One of the critical goals is to be able to generate 3D structures from a set of images that can be done in either in a semi-automated manner in a high-throughput manner. As a result, we have incorporated 
development of programs and procedures within the EMAN package, a well-known package for single particle reconstruction [10]. We have developed a much improved version for correlation averaging using a program called QINDEX. This program displays almost real time diffraction patterns of sub-areas, calculates a lattice filtered reference image, calculates the cross-correlation map, selects the highest correlating areas and finally, writes out the coordinates of those areas for boxing by the EMAN program, BOXER. Since the local lattice serves to physically translationally align the channels, boxed particles can simply be averaged and then treated as class averages in a traditional single particle reconstruction scheme. A flowchart and sample results is shown in Fig. 1.

\section{$\underline{\text { References }}$}

[1] Sosinsky, G.E. and B.J. Nicholson, Biochim Biophys Acta, 2005. 1711: 99.

[2] Unger, V.M., et al., Science, 1999. 283: 1176.

[3] Oshima, A., et al., Microscopy and Microanalysis, 2006. Supplement: in press.

[4] Beahm, D., et al., J.Biol.Chem., 2005. in press.

[5] Muller, D.J., et al., EMBO J, 2002. 21: 3598.

[6] Lawrence, A., et al., J Struct. Biol., 2006: in press.

[7] Sosinsky, G.E., et al., Biophys. J., 1990. 58: 1213.

[8] Saxton, W.O., W. Baumeister, and M. Hahn, Ultramicrosc., 1984. 13: 57.

[9] Perkins, G.A., D.A. Goodenough, and G.E. Sosinsky, Biophys. J., 1997. 72: 533.

[10] Ludtke, S.J., P.R. Baldwin, and W. Chiu, J Struct Biol, 1999. 128: 82.

[11] This work is supported by NIH grants GM065937 (GS), RR04050 (NCMIR, Mark Ellisman), NSF MCB-0131425 (GS) and RR02250 (NCMI, Wah Chiu).
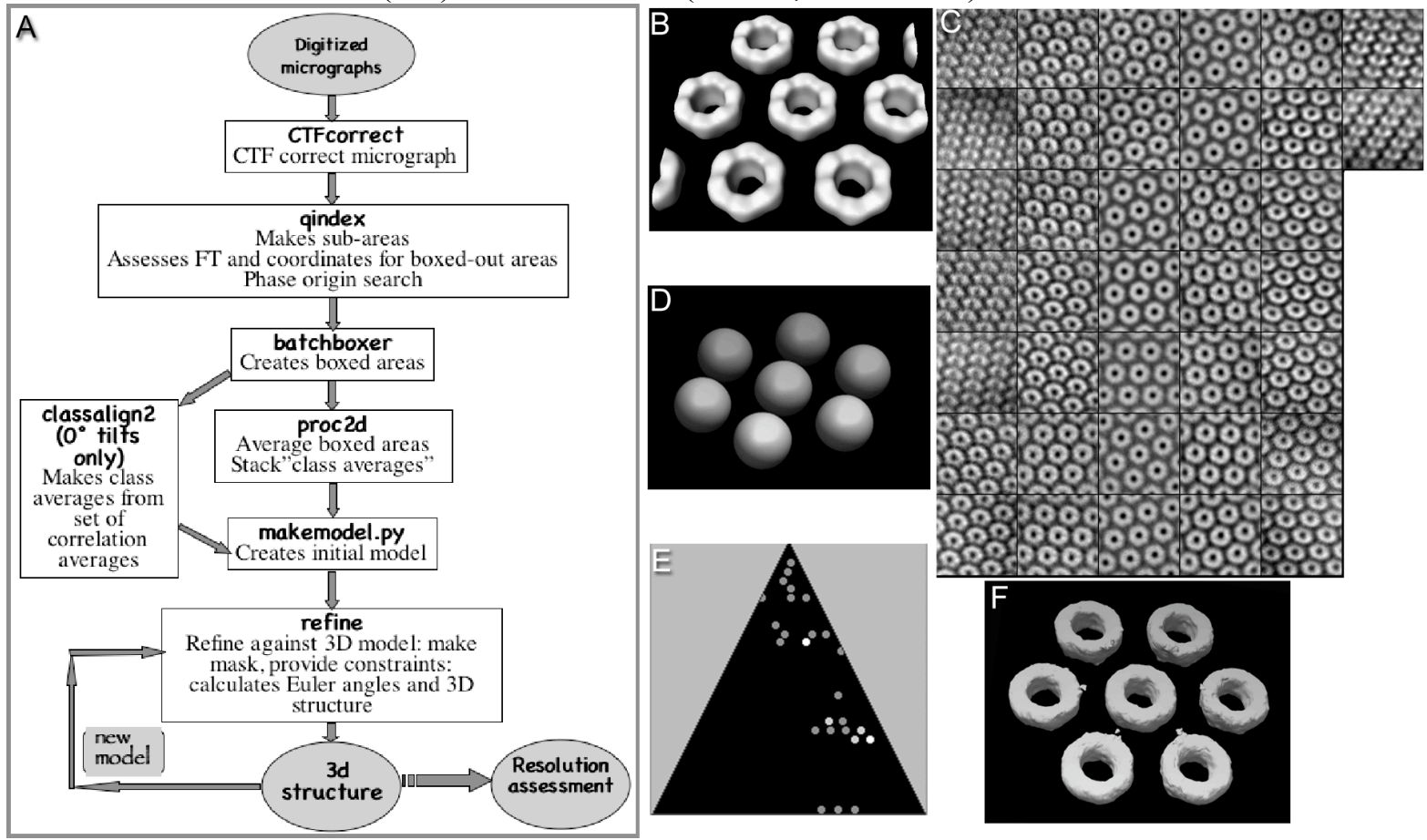

Fig. 1 (A) Flowchart illustrating steps and EMAN programs used in this new 3D reconstruction schema. (B) Crystallographically derived 3D structure from this sample data set [9]. (C) Set of "class averages" from correlation averaging. (D) Initial model for alignment. (E) Distribution of Euler angles in this data set. (F) Sample 3D reconstruction using this procedure. 